\title{
Fractal mechanisms of light scattering in biological tissue and cells
}

Min Xu

Fairfield University, mxu@fairfield.edu

R. R. Alfano

Follow this and additional works at: https://digitalcommons.fairfield.edu/physics-facultypubs

(C) 2005 The Optical Society. This paper was published in Optics letters and is made available as an electronic reprint with the permission of OSA. The paper can be found at the following URL on the OSA website: http://www.opticsinfobase.org/ol/abstract.cfm?URI=ol-30-22-3051 Systematic or multiple reproduction or distribution to multiple locations via electronic or other means is prohibited and is subject to penalties under law.

\section{Peer Reviewed}

\section{Repository Citation}

$\mathrm{Xu}, \mathrm{Min}$ and Alfano, R. R., "Fractal mechanisms of light scattering in biological tissue and cells" (2005).

Physics Faculty Publications. 11.

https://digitalcommons.fairfield.edu/physics-facultypubs/11

\section{Published Citation}

M. Xu and R. R. Alfano. Fractal mechanisms of light scattering in biological tissue and cells. Optics Letters, 30(22):3051-3053, 2005. http://www.opticsinfobase.org/ol/abstract.cfm?URI=ol-30-22-3051

This item has been accepted for inclusion in DigitalCommons@Fairfield by an authorized administrator of DigitalCommons@Fairfield. It is brought to you by DigitalCommons@Fairfield with permission from the rightsholder(s) and is protected by copyright and/or related rights. You are free to use this item in any way that is permitted by the copyright and related rights legislation that applies to your use. For other uses, you need to obtain permission from the rights-holder(s) directly, unless additional rights are indicated by a Creative Commons license in the record and/or on the work itself. For more information, please contact digitalcommons@fairfield.edu. 


\title{
Fractal mechanisms of light scattering in biological tissue and cells
}

\author{
M. Xu and R. R. Alfano \\ Institute for Ultrafast Spectroscopy and Lasers, and Department of Physics, The City College and Graduate Center \\ of City University of New York, New York, New York 10031
}

Received April 21, 2005; revised manuscript received June 22, 2005; accepted June 30, 2005

We use fractal continuous random media to model visible and near-infrared light scattering by biological tissue and cell suspensions. The power law of the reduced scattering coefficient, the anisotropy factor of scattering, and the phase function are derived with good agreement with experimental results. Implications for spectroscopic tissue diagnosis are discussed. (C) 2005 Optical Society of America

OCIS codes: $170.3660,170.4580,170.6510,170.1530,290.0290$.

The interaction of light with tissue and cells is the underlying mechanism for optical biomedical technology used in optical imaging and spectroscopy for detection of pathology changes. The optical properties of tissue are determined by chromophores, microstructures, and local refractive index variations. Microstructures in biological tissue range from organelles $0.2-0.5 \mu \mathrm{m}$ or smaller, to mitochondria $1-4 \mu \mathrm{m}$ in length and $0.3-0.7 \mu \mathrm{m}$ in diameter, to nuclei $3-10 \mu \mathrm{m}$ in diameter, to mammalian cells $10-30 \mu \mathrm{m}$ in diameter. The refractive index variation is about $0.04-0.10$ for biological tissue with a background refractive index of $n_{0}=1.35 .^{1}$ Recently, the nature of light scattering in biological tissue has been actively studied. ${ }^{1-5}$ The fractal dimension of a monolayer of cells was investigated. ${ }^{6,7}$ As many biological tissues have fractallike organization and are statistically self-similar, ${ }^{2,8}$ a discrete particle model of scattering centers in tissue ${ }^{1,4}$ was proposed to model light scattering by tissue. The discrete particle model assumes that the refractive index variations caused by underlying microscopic structures can be treated as spherical particles with sizes distributed according to a power law:

$$
\eta(a)=\eta_{0} a^{3-D_{f}},
$$

where $\eta(a)$ is the volume fraction of spherical particles of radius $0 \leqslant a \leqslant a_{\max }$ with a maximum radius $a_{\max }, \eta_{0}$ is a constant, and $D_{f}$ is the fractal dimension. On a microscopic scale the constituents of tissue have no clear boundaries and merge into a quasicontinuum structure. Discrete particles may not be appropriate to describe tissue inhomogeneities. As the refractive index variation in biological tissue is weak, tissue is better modeled as a continuous random medium where light scattering is not due to the discontinuities in refractive index but rather to weak random fluctuations of the dielectric permittivity. ${ }^{5}$

In this Letter, we propose to use fractal continuous random media to model light scattering by biological tissue and cells. The correlation function $R(r)$ of the random fluctuations of the dielectric permittivity depends on the fractal dimension $D_{f}$ and the cutoff correlation length $l_{\text {max }}$. Analytical expressions are derived for the power law of the reduced scattering coefficient, the anisotropy factor of scattering, and the phase function. By examination of the existing experimental results, the fractal fluctuation of the dielectric permittivity is shown to determine visible and near-infrared light scattering by biological tissue and cell suspensions.

Assuming that tissue is statistically space homogeneous and isotropic, the correlation function of the dielectric permittivity can be written as $R(r)$ $=\left\langle\delta \epsilon\left(\mathbf{r}^{\prime}\right) \delta \epsilon\left(\mathbf{r}^{\prime}+\mathbf{r}\right)\right\rangle$, where $\delta \epsilon$ is the fluctuation of the dielectric permittivity from the background value. $R(\mathbf{r})$ is proportional to the correlation function of the fluctuation of the refractive index $R_{n}(\mathbf{r})$ and $\delta \epsilon(\mathbf{r})$ $=2 n_{0}^{2}(m-1)$, where $m$ is the relative refractive index at position $\mathbf{r}$ when the fluctuation of the refractive index is weak. Light scattering by the continuous random medium is determined by the power spectrum $\hat{R}$ of the random fluctuations of the dielectric permittivity. The amplitude scattering matrix of the weakly fluctuating continuous random medium at the scattering angle $\theta$ is given by

$$
S(\theta)\left[\begin{array}{cc}
\cos \theta & 0 \\
0 & 1
\end{array}\right]=k^{3} \sqrt{\frac{\pi}{2} \hat{R}\left(2 k \sin \frac{\theta}{2}\right)}\left[\begin{array}{cc}
\cos \theta & 0 \\
0 & 1
\end{array}\right],
$$

where $k \equiv 2 \pi n_{0} / \lambda$ is the wavenumber and $\lambda$ is the wavelength of light in vacuum. A simple exponential correlation function was considered by Moscoso et al. ${ }^{5}$ for modeling tissue light scattering.

The correlation function of the random fluctuations of the dielectric permittivity, in the fractal continuous medium model, is assumed to be an average of exponential functions weighted by power law distribution (1) for correlation length $l$ :

$R(r)=\varepsilon^{2} \int_{0}^{l_{\max }} \exp \left(-\frac{r}{l}\right) \eta(l) \mathrm{d} l=\varepsilon^{2} \eta_{0} l_{\max }^{4-D_{f}} E_{5-D_{f}}\left(\frac{r}{l_{\max }}\right)$

where $\varepsilon^{2}=4 n_{0}^{4}(m-1)^{2}, l_{\max }$ is the cutoff correlation length, and $E_{n}(z) \equiv \int_{0}^{1} \exp (-z / t) t^{n-2} \mathrm{~d} t$. Correlation function (3) is adopted based on the observed similarity of the refractive index fluctuation of biological tissue and inspired by the discrete particle model. ${ }^{1,4}$ 
The correlation function at the origin is $R(0)=\varepsilon^{2} T_{v}$, where $T_{v}$ is the total volume fraction of scattering centers $\left(T_{v} \simeq 0.3\right.$ in soft tissue). The correlation function decays exponentially as $R(r)$ $=\varepsilon^{2} \eta_{0} l_{\max }^{4-D_{f}}\left(r / l_{\max }\right)^{-1} \exp \left(-r / l_{\max }\right)$ at a large separation $r \gg l_{\max }$. The power spectrum is given by

$$
\hat{R}(k)=\int_{0}^{l_{\max }} \frac{\varepsilon^{2} l^{3}}{\pi^{2}\left(1+k^{2} l^{2}\right)^{2}} \eta(l) \mathrm{d} l .
$$

The amplitude scattering function $S(\theta)$ in Eq. (2) can now be written as

$$
|S(\theta)|^{2}=\int_{0}^{k l_{\max }} \frac{\varepsilon^{2} \eta_{0} k^{D_{f-1}} x^{6-D_{f}}}{2 \pi\left[1+2(1-\mu) x^{2}\right]^{2}} \mathrm{~d} x,
$$

where $\mu \equiv \cos \theta$. The anisotropy factor (mean cosine of the scattering angle) can be found:

$$
g=\int \mathrm{d} \Omega \frac{\left(1+\mu^{2}\right) \mu|S(\theta)|^{2}}{2 k^{2}} / \int \mathrm{d} \Omega \frac{\left(1+\mu^{2}\right)|S(\theta)|^{2}}{2 k^{2}} .
$$

The unnormalized phase function is given by

$$
\begin{aligned}
p(\theta) & =\frac{\left(1+\mu^{2}\right)|S(\theta)|^{2}}{2 k^{2}} \\
& =\frac{\frac{5-D_{f}}{2} \pi}{\sin \left(\frac{5-D_{f}}{2} \pi\right)} \frac{1+\mu^{2}}{2}[2(1-\mu)]^{-\left(7-D_{f}\right) / 2} \\
\propto & \left(1+\mu^{2}\right)(1-\mu)^{-\left(7-D_{f}\right) / 2} .
\end{aligned}
$$

The reduced scattering coefficient, defined as $\mu_{s}(1$ $-g)$, is given by

$$
\mu_{s}^{\prime}=\int \mathrm{d} \Omega \frac{\left(1+\mu^{2}\right)(1-\mu)|S(\theta)|^{2}}{2 k^{2}} \simeq \varepsilon^{2} \eta_{0} \alpha \lambda^{3-D_{f}},
$$

where

$$
\begin{gathered}
\alpha \equiv\left(2 \pi n_{0}\right)^{D_{f}-3} \frac{\left(11-4 D_{f}+D_{f}^{2}\right)}{\left(D_{f}+1\right)\left(D_{f}-1\right)\left(D_{f}-3\right)} \\
\times \frac{\frac{5-D_{f}}{2} \pi}{2^{5-D_{f}} \sin \left(\frac{5-D_{f}}{2} \pi\right)}
\end{gathered}
$$

is a constant dependent only on $D_{f}$. We have assumed that $k l_{\max } \gg 1$ in our derivations of Eqs. (7) and (8).

The values of $g$ in the fractal continuous medium model versus the cutoff size parameter $k l_{\max }$ for various $D_{f}$ are displayed in Fig. 1(a). $g$ is larger with the increase of $l_{\max }$ and the decrease of $D_{f}$. Light scattering equation (5) can be regarded as a weighted sum from components of different correlation lengths where the peak contribution occurs at $k l^{*}=k l_{\text {max }}$ when $D_{f}<4$ and $k l^{*}$ may be different from $k l_{\max }$ when $D_{f}>4$. The value of $k l^{*}$ when $D_{f}>4$ is displayed in Fig. 1(b). The power law of $\mu_{s}^{\prime}$, Eq. (8), is obtained due to the cutoff at large correlation lengths as $g$ approaches unity for components of increasing correlation lengths. By fitting the experimental wavelength dependence of $\mu_{s}^{\prime}$ to Eq. (8), one can determine both the fractal dimension $D_{f}$ and the parameter $\varepsilon^{2} \eta_{0}$.

We first fit the theoretical power spectrum, Eq. (4), to the power spectrum of index variations in mouse liver tissue reported by Schmitt et al. ${ }^{2}$ [see Fig. 2(a)]. The fit is excellent and yields $l_{\max }=2.3 \mu \mathrm{m}$ and $D_{f}$ $=4.0$ for mouse liver tissue. A single exponential correlation function ${ }^{5}$ will not fit. We do not know any results on the wavelength dependence of the reduced scattering coefficient for mouse liver tissue. The wavelength dependence of the reduced scattering coefficient of rat liver tissue was reported by Parsa $e t$ $a l .{ }^{9}$ The reduced scattering coefficients within the range 600 to $1400 \mathrm{~nm}$ are displayed in Fig. 2(b) and fitted well to a power law $\lambda^{-0.94}$. The fractal dimension of rat liver tissue is hence $D_{f}=3.94$. The cutoff correlation length is found to be $l_{\max }=1.5 \mu \mathrm{m}$ from $g=0.94$ of the rat liver tissue at $800 \mathrm{~nm}$. Evidently, both the fractal dimension and the cutoff correlation length extracted from the power spectrum of the refractive index variations of mouse liver tissue agree
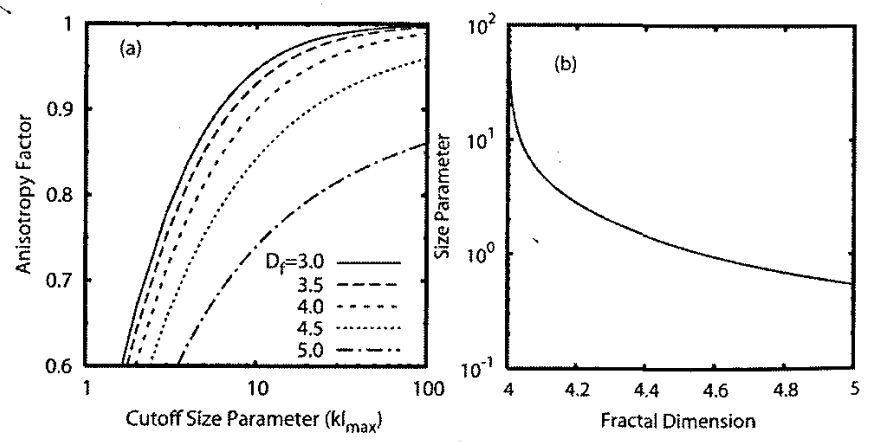

Fig. 1. (a) Anisotropy factor of the fractal continuous random medium versus cutoff size parameter $k l_{\max }$ (b) Size parameter $k l^{*}$ of the component contributing most to light scattering when $D_{f}>4$.
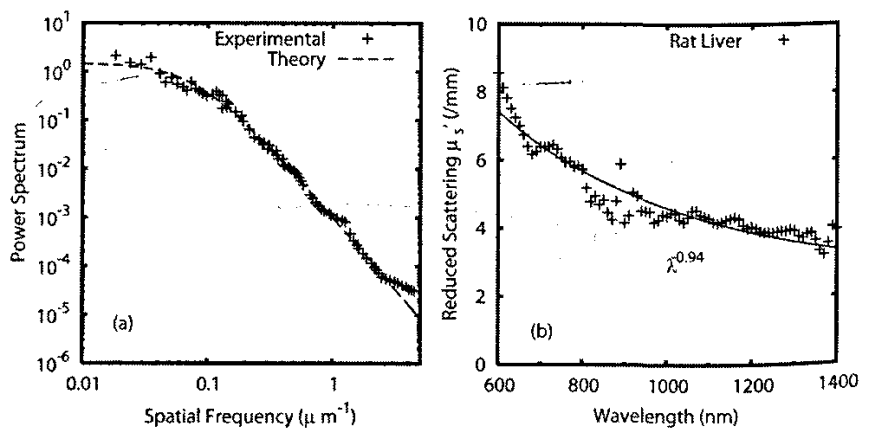

Fig. 2. (a) Power spectrum of refractive index variations in mouse liver tissue fitted to the theoretical power spectrum, Eq. (4). The symbols represent data reported in Ref. 2 , and the dashed curve is the theoretical fit. Fitting yields $D_{f}=4.0$ and $l_{\max }=2.3 \mu \mathrm{m}$. (b) Wavelength dependence of the reduced scattering coefficient of rat liver tissue fitted to the power law $\lambda^{3-D_{f}}$. The symbols represent data reported in Ref. 9, and the solid curve shows the fitted curve. Fitting yields $D_{f}=3.94$ and $l_{\max }=1.5 \mu \mathrm{m}$. 
reasonably well with those extracted from the lightscattering data for rat liver tissue.

Figure 3 displays the phase functions for suspensions of rat embryo fibroblast cells (M1) and mitochondria reported by Mourant et al. ${ }^{3}$ and the fit to the theoretical phase function (7). The fractal dimension is found to be $D_{f}=3.86$ and 4.58 for M1 cells and mitochondria, respectively. The value of the fractal dimension for M1 cells was $4 \pm 0.07$ with the reported wavelength dependence $\mu_{s}^{\prime} \propto \lambda^{-1.0 \pm 0.07}$ over the wavelength range $500-800 \mathrm{~nm}$. The agreement between the two values of $D_{f}$ for M1 cells from fitting either the phase function or the power law of $\mu_{s}^{\prime}$ is good. The $g$ factor for M1 cells was reported to be 0.98 , and the maximum correlation length can be estimated to be $l_{\max } \sim 3.2 \mu \mathrm{m}$. The $g$ factor for mitochondria can be computed from the phase function to be 0.81 , and the maximum correlation length is then estimated to be $l_{\max } \sim 0.6 \mu \mathrm{m}$. The component contributing the most to light scattering has size parameter $k l^{*}=k l_{\max } \simeq 40$ and $k l^{*} \simeq 1$ in M1 cells and mitochondria, respectively. M1 cells have much larger scattering centers and a much smaller fractal dimension than mitochondria. The larger scattering centers in M1 cells are due to the nucleus.

The power in the power law of $\mu_{s}^{\prime} \propto \lambda^{-b}$ is usually called the scattering power. The scattering power in the fractal continuous medium model relates to the fractal dimension of the underlying fluctuation of the refractive index $\left(b=D_{f}-3\right)$ and should be distinguished from that due to Mie particles of narrow size distribution. ${ }^{3}$ The scattering power has been recognized to be an important parameter in discriminating normal and cancerous tissue. ${ }^{10-12}$ Both the fractal di-

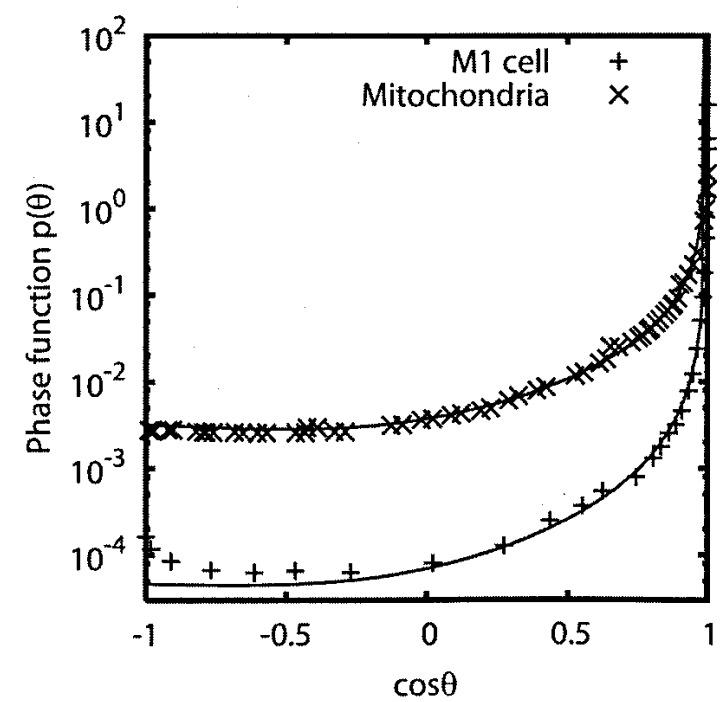

Fig. 3. Phase function of suspensions of rat embryo fibroblast cells (M1) and mitochondria fitted to theoretical phase function (7). The symbols are data reported in Ref. 3, and the solid curves are theoretical curves. Fitting yields $D_{f}=3.86$. and 4.58 for M1 cells and mitochondria, respectively. mension $D_{f}$ and the parameter $\varepsilon^{2} \eta_{0}$ can be estimated from fitting the wavelength dependence of $\mu_{s}^{\prime}$ to Eq. (8) in the fractal continuous medium model. The value of $D_{f}$ reveals the relative weight of small scattering centers versus large scattering centers. The value of $\varepsilon^{2} \eta_{0}$ represents the overall density of scattering centers, which is proportional to the radiographic density of tissue of predictive value for cancer risk. ${ }^{13}$ The maximum correlation length $l_{\max }$ can be estimated from the anisotropy factor. The access to all these parameters will yield much more valuable information about the structure and the physiological state of tissue than using the scattering power alone.

In conclusion, we have shown that light-scattering properties of biological tissue and cell suspensions can be well represented by a fractal continuous random medium model where light scattering is due to weak random fluctuations of the dielectric permittivity. The fractal dimension $D_{f}$ and the cutoff correlation length $l_{\max }$ characterizes the essential features of light scattering by such media. The fractal continuous random medium model should facilitate the analysis of light-scattering spectroscopy for tissue diagnosis.

This work was supported in part by NASA and the Department of the Army (grant DAMD1702-1-0516). M. Xu's e-mail address is minxu@sci.ccny.cuny.edu.

\section{References}

1. J. M. Schmitt and G. Kumar, Appl. Opt. 37, 2788 (1998).

2. J. M. Schmitt and G. Kumar, Opt. Lett. 21, 1310 (1996).

3. J. R. Mourant, J. P. Freyer, A. H. Hielscher, A. A. Eick, D. Shen, and T. M. Johnson, Appl. Opt. 37, 3586 (1998).

4. R. K. Wang, J. Mod. Opt. 47, 103 (2000).

5. M. Moscoso, J. B. Keller, and G. Papanicolaou, J. Opt. Soc. Am. A 18, 948 (2001).

6. V. Backman, V. Gopal, M. Kalashnikov, K. Badizadegan, R. Gurjar, A. Wax, I. Georgakoudi, M. Mueller, C. W. Boone, R. R. Dasari, and M. S. Feld, IEEE J. Sel. Top. Quantum Electron. 7, 887 (2001).

7. A. Wax, C. Yang, V. Backman, K. Badizadegan, C. W. Boone, R. R. Dasari, and M. S. Feld, Biophys. J. 82, 2256 (2002).

8. A. J. Einstein, H.-S. Wu, and J. Gil, Phys. Rev. Lett. 80, 397 (1998).

9. P. Parsa, S. L. Jacques, and N. S. Nishioka, Appl. Opt. 28, 2325 (1989).

10. N. Ghosh, S. K. Mohanty, S. K. Majumder, and P. K. Gupta, Appl. Opt. 40, 176 (2001).

11. A. E. Cerussi, A. J. Berger, F. Bevilacqua, N. Shah, D. Jakubowski, J. Butter, R. F. Holcombe, and B. J. Tromberg, Acad. Radiol. 8, 211 (2001).

12. A. Pifferi, J. Swartling, E. Chikoidze, A. Torricelli, P. Taroni, A. Bassi, S. Andersson-Engels, and R. Cubeddu, J. Biomed. Opt. 9, 1143 (2004).

13. K. Blyschak, M. Simick, R. Jong, and L. Lilge, Med. Phys. 31, 1398 (2004). 